\title{
A Note on Symplectic, Multisymplectic Scheme in Finite Element Method
}

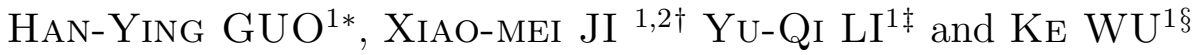 \\ 1 Institute of Theoretical Physics, Academia Sinica, P.O.Box 2735, \\ Beijing 100080, China \\ 2 Department of Mathematics, Indiana University \\ Bloomington, IN 47405, U.S.A.
}

\begin{abstract}
We find that with uniform mesh, the numerical schemes derived from finite element method can keep a preserved symplectic structure in one-dimensional case and a preserved multisymplectic structure in two-dimentional case in certain discrete version respectively. These results are in fact the intrinsic reason that the numerical experiments indicate that such finite element algorithms are accurate in practice.
\end{abstract}

Keywords: symplectic structure, finite element method, numerical scheme

*email: hyguo@itp.ac.cn

†email: jixm@iu-math.math.indiana.edu

‡email: qylee@itp.ac.cn

§email: wuke@itp.ac.cn 
As both the finite elemente method[1] and symplectic scheme [2] as well as multisymplectic scheme [3] are powerful tools to solve differential equations numericaly, it is interesting to explore if there is some relation between them. We will give a partial answer to this question by a simple example.

In order to show the symplectic or multisymplectic structures in the scheme derived from finite elemente method, we consider the boundary value problem of the semilinear elliptic equation in one-dimensional and two-dimensional spaces:

$$
\triangle u=f(u) \text { in } \Omega,\left.\quad u\right|_{\partial \Omega}=0 \quad \text { on } \quad \partial \Omega .
$$

where $\Omega$ is a bounded domain in $\mathbb{R}^{n}, n=1,2$ and $f(u)$ is nonlinear and sufficiently smooth enough function. The weak formulation of the boundary value problem of the equation is to find $u: \Omega \rightarrow H_{0}^{1}(\Omega)$ such that

$$
\int_{\Omega} \nabla u \cdot \nabla v d x=-\int_{\Omega} f \cdot v d x \quad \forall v \in H_{0}^{1}(\Omega)
$$

Let

$$
a(u, v)=\int_{\Omega} \nabla u \cdot \nabla v d x, \quad(f, v)=-\int_{\Omega} f \cdot v d x
$$

then (2) becomes,

$$
a(u, v)=(f, v)
$$

It is important to note that the equation is in fact an ODE with Lagrangian on $\mathbb{R}^{1}$ or Lagrangian PDE in $\mathbb{R}^{2}$ respectively. The details of the symplectic and multisymplectic structures in the Lagrangian formalism can be found in [2] |3] [4] [5] [6] [7] [8].

In one-dimensional case we first discrete $\mathbb{R}^{1}$ with regular lattice $\mathbb{L}^{1}$ with equal spatial step $h$

$$
\mathbb{L}^{1}=\left\{\cdots, x_{i-1}, x_{i}, x_{i+1}, \cdots\right\}
$$

Let $\Omega$ be a segment in $\mathbb{R}^{1}$ and $\varphi_{i}$ linear shape function such that $\varphi_{i}\left(x_{j}\right)=\delta_{i j}$. As usual, $u_{i}=u\left(x_{i}\right)$.

From the finite element method, we get the scheme

$$
\frac{u_{i+1}-2 u_{i}+u_{i-1}}{h}=\int_{i-1}^{i+1} f\left(\sum_{i-1}^{i+1} u_{k} \varphi_{k}\right) \varphi_{i} d x .
$$

The right side can be rewritten as

$$
I_{i}:=\int_{i-1}^{i} f\left(u_{i-1} \varphi_{i-1}+u_{i} \varphi_{i}\right) \varphi_{i} d x+\int_{i}^{i+1} f\left(u_{i} \varphi_{i}+u_{i+1} \varphi_{i+1}\right) \varphi_{i} d x
$$

It should be noted that the variables $u_{i}$ 's in the scheme can be released from the solution space to the function space by means of relevant discrete Euler-Lagrange (DEL) cohomologically equivalent relation [4]. Therefore, as long as working with the DEL cohomology class associated with the DEL euation (times by certain 1-form) the $u_{k}$ 's can be regarded as in the function space in general rather than in the solution space.

Introducing the DEL 1-forms

$$
E_{D i}:=\left\{u_{i+1}-2 u_{i}+u_{i-1}-h I_{i}\right\} d u_{i}
$$


such that the null DEL 1-form gives rise to the equation in the finite element method. The DEL condition reads

$$
d E_{D i}=0
$$

Namely, the DEL 1-forms are closed. It is straightforward to see that from the DEL condition it follows

$$
\begin{aligned}
& d u_{i+1} \wedge d u_{i}+d u_{i-1} \wedge d u_{i} \\
= & h\left(\int_{i-1}^{i} f^{\prime}\left(u_{i-1} \varphi_{i-1}+u_{i} \varphi_{i}\right) \varphi_{i-1} \varphi_{i} d x\right) d u_{i-1} \wedge d u_{i} \\
+ & h\left(\int_{i}^{i+1} f^{\prime}\left(u_{i} \varphi_{i}+u_{i+1} \varphi_{i+1}\right) \varphi_{i+1} \varphi_{i} d x\right) d u_{i+1} \wedge d u_{i}
\end{aligned}
$$

i.e.,

$$
\omega^{(i+1)}=\omega^{(i)}
$$

where

$$
\omega^{(i+1)}=\left(1-h \int_{i}^{i+1} f^{\prime}\left(u_{i} \varphi_{i}+u_{i+1} \varphi_{i+1}\right) \varphi_{i+1} \varphi_{i} d x\right) d u_{i+1} \wedge d u_{i}
$$

It can be checked that this 2 -form is closed w.r.t. $d$ on the function space and its coefficients are non-degenerate, so that it is a symplectic structure for the scheme derived from finite element method in one-dimensional and it is preserved.

In the two-dimensional case, the semi-linear equation becomes

$$
u_{x_{1} x_{1}}+u_{x_{2} x_{2}}=f(u) \quad \text { in } \quad \Omega,\left.\quad u\right|_{\partial \Omega}=0 \quad \text { on } \quad \partial \Omega
$$

where $u_{x_{i}}, i=1,2$, denote the partial derivative of $u$ w.r.t. coordinate $\left\{x_{i}\right\}$ in $\mathbb{R}^{2}$. Without loss of generality, we assume $\Omega$ is a square domain, and the mesh is uniform, that is, the plane $\mathbb{R}^{2}$ is divided into squares $\left\{\left(x_{1}, x_{2}\right) ; i_{1} h \leq x_{1} \leq\left(i_{1}+1\right) h, i_{2} h \leq x_{2} \leq\right.$ $\left.\left(i_{2}+1\right) h\right\}, i_{1}, i_{2}=0, \pm 1, \pm 2, \cdots$, and each square is further divided into two triangles by a straight line $x_{2}=x_{1}+i h, i$ integer. Take the node $x_{i, j}$ and $\Omega_{i, j}$ shown as figure 1. The elements are divided into two categories: the first category is shown as figure 2 and the second is shown as figure 3 .

The discrete scheme for this equation form finite element method is

$$
\begin{array}{r}
u_{i, j+1}+u_{i+1, j}+u_{i, j-1}+u_{i-1, j}-4 u_{i, j} \\
=\int_{\Omega_{i, j}} f\left(\sum u_{k, l} \varphi_{k, l}\right) \varphi_{i, j} d x
\end{array}
$$

where the shape function $\varphi_{i, j}$ is linear continuous function defined as

$$
\varphi_{i, j}\left(x_{k, l}\right)=\delta_{i . k} \delta_{j, l}
$$

The right side of (12) is

$$
\begin{aligned}
I_{\Omega_{i, j}} & :=\int_{\Omega_{i, j}} f\left(\sum u_{k, l} \varphi_{k, l}\right) \varphi_{i, j} d x \\
& =\left(\int_{A}+\int_{B}+\int_{C}+\int_{D}+\int_{E}+\int_{F}\right) f\left(\sum u_{k, l} \varphi_{k, l}\right) \varphi_{i, j} d x,
\end{aligned}
$$




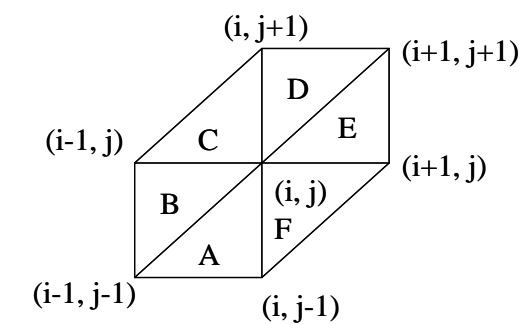

Figure 1.

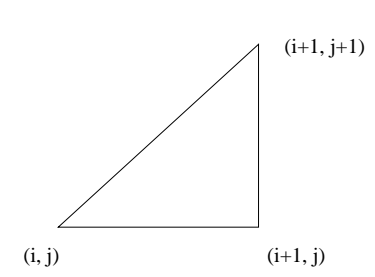

Figure 2.

Figure 1:

Figure 2:

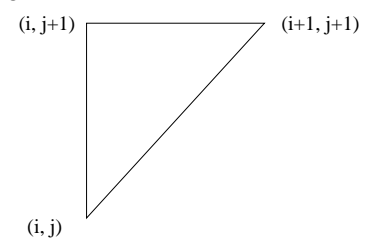

Figure 3.

Figure 3:

where

$$
\begin{aligned}
& I_{\Omega_{i, j} A}: \int_{A} f\left(\sum u_{k, l} \varphi_{k, l}\right) \varphi_{i, j} d x \\
&= \int_{A} f\left(u_{i-1, j-1} \varphi_{i-1, j-1}+u_{i, j-1} \varphi_{i, j-1}+u_{i, j} \varphi_{i, j}\right) \varphi_{i, j} d x \\
& I_{\Omega_{i, j} B}:=\int_{B} f\left(\sum u_{k, l} \varphi_{k, l}\right) \varphi_{i, j} d x \\
&=\int_{B} f\left(u_{i-1, j-1} \varphi_{i-1, j-1}+u_{i-1, j} \varphi_{i-1, j}+u_{i, j} \varphi_{i, j}\right) \varphi_{i, j} d x \\
& I_{\Omega_{i, j} C}:=\int_{C} f\left(\sum u_{k, l} \varphi_{k, l}\right) \varphi_{i, j} d x \\
&=\int_{C} f\left(u_{i-1, j} \varphi_{i-1, j}+u_{i, j} \varphi_{i, j}+u_{i, j+1} \varphi_{i, j+1}\right) \varphi_{i, j} d x \\
& I_{\Omega_{i, j} D}:= \int_{D} f\left(\sum u_{k, l} \varphi_{k, l}\right) \varphi_{i, j} d x \\
&= \int_{D} f\left(u_{i+1, j+1} \varphi_{i+1, j+1}+u_{i, j} \varphi_{i, j}+u_{i, j+1} \varphi_{i, j+1}\right) \varphi_{i, j} d x \\
& I_{\Omega_{i, j} E}:= \int_{E} f\left(\sum u_{k, l} \varphi_{k, l}\right) \varphi_{i, j} d x \\
&= \int_{E} f\left(u_{i+1, j+1} \varphi_{i+1, j+1}+u_{i, j} \varphi_{i, j}+u_{i+1, j} \varphi_{i+1, j}\right) \varphi_{i, j} d x \\
&=\int_{F} f\left(u_{i+1, j} \varphi_{i+1, j}+u_{i, j} \varphi_{i, j}+u_{i, j-1} \varphi_{i, j-1}\right) \varphi_{i, j} d x . \\
&\left.I_{k, l}\right) \varphi_{i, j} d x \\
&=
\end{aligned}
$$


where the sub-index $A, B, C, D, E, F$ indicate the all elements neighboring $x_{i, j}$ in Figure 1.

Similar to the one-dimensional case, introducing the DEL 1-forms

$$
E_{D \Omega_{i, j}}:=\left\{u_{i, j+1}+u_{i+1, j}+u_{i, j-1}+u_{i-1, j}-4 u_{i, j}-I_{\Omega_{i, j}}\right\} d u_{i, j} .
$$

The DEL condition now reads

$$
d E_{D \Omega_{i, j}}=0
$$

i.e.

$$
\begin{aligned}
& d u_{i, j+1} \wedge d u_{i, j}+d u_{i+1, j} \wedge d u_{i, j}+d u_{i, j-1} \wedge d u_{i, j}+d u_{i-1, j} \wedge d u_{i, j} \\
& =S_{A\{i, j\}}+S_{B\{i, j\}}+S_{C\{i, j\}}+S_{D\{i, j\}}+S_{E\{i, j\}}+S_{F\{i, j\}}
\end{aligned}
$$

where,

$$
\begin{aligned}
S_{A\{i, j\}}= & \left(\int_{D} f^{\prime}\left(u_{i+1, j+1} \varphi_{i+1, j+1}+u_{i, j} \varphi_{i, j}+u_{i, j+1} \varphi_{i, j+1}\right) \varphi_{i+1, j+1} \varphi_{i, j} d x+\right. \\
& \left.\int_{E} f^{\prime}\left(u_{i+1, j+1} \varphi_{i+1, j+1}+u_{i, j} \varphi_{i, j}+u_{i+1, j} \varphi_{i+1, j}\right) \varphi_{i+1, j+1} \varphi_{i, j} d x\right) \cdot d u_{i+1, j+1} \wedge d u_{i, j}, \\
S_{B\{i, j\}}= & \left(\int_{D} f^{\prime}\left(u_{i+1, j+1} \varphi_{i+1, j+1}+u_{i, j} \varphi_{i, j}+u_{i, j+1} \varphi_{i, j+1}\right) \varphi_{i, j+1} \varphi_{i, j} d x+\right. \\
& \left.\int_{C} f^{\prime}\left(u_{i-1, j} \varphi_{i-1, j}+u_{i, j} \varphi_{i, j}+u_{i, j+1} \varphi_{i, j+1}\right) \varphi_{i, j+1} \varphi_{i, j} d x\right) \cdot d u_{i, j+1} \wedge d u_{i, j}, \\
S_{C\{i, j\}}= & \left(\int_{E} f^{\prime}\left(u_{i+1, j+1} \varphi_{i+1, j+1}+u_{i, j} \varphi_{i, j}+u_{i+1, j} \varphi_{i+1, j}\right) \varphi_{i+1, j} \varphi_{i, j} d x+\right. \\
& \left.\int_{F} f^{\prime}\left(u_{i+1, j} \varphi_{i+1, j}+u_{i, j} \varphi_{i, j}+u_{i, j-1} \varphi_{i, j-1}\right) \varphi_{i+1, j} \varphi_{i, j} d x\right) \cdot d u_{i+1, j} \wedge d u_{i, j}, \\
S_{D\{i, j\}}= & \left(\int_{F} f^{\prime}\left(u_{i+1, j} \varphi_{i+1, j}+u_{i, j} \varphi_{i, j}+u_{i, j-1} \varphi_{i, j-1}\right) \varphi_{i, j-1} \varphi_{i, j} d x+\right. \\
& \left.\int_{A} f^{\prime}\left(u_{i-1, j-1} \varphi_{i-1, j-1}+u_{i, j-1} \varphi_{i, j-1}+u_{i, j} \varphi_{i, j}\right) \varphi_{i, j-1} \varphi_{i, j} d x\right) \cdot d u_{i, j-1} \wedge d u_{i, j}, \\
S_{E\{i, j\}}= & \left(\int_{A} f^{\prime}\left(u_{i-1, j-1} \varphi_{i-1, j-1}+u_{i, j-1} \varphi_{i, j-1}+u_{i, j} \varphi_{i, j}\right) \varphi_{i-1, j-1} \varphi_{i, j} d x+\right. \\
& \left.\int_{B} f^{\prime}\left(u_{i-1, j-1} \varphi_{i-1, j-1}+u_{i-1, j} \varphi_{i-1, j}+u_{i, j} \varphi_{i, j}\right) \varphi_{i-1, j-1} \varphi_{i, j} d x\right) \cdot d u_{i-1, j-1} \wedge d u_{i, j}, \\
= & \left(\int_{B} f^{\prime}\left(u_{i-1, j-1} \varphi_{i-1, j-1}+u_{i-1, j} \varphi_{i-1, j}+u_{i, j} \varphi_{i, j}\right) \varphi_{i-1, j} \varphi_{i, j} d x+\right. \\
& \left.\int_{C} f^{\prime}\left(u_{i-1, j} \varphi_{i-1, j}+u_{i, j} \varphi_{i, j}+u_{i, j+1} \varphi_{i, j+1}\right) \varphi_{i-1, j} \varphi_{i, j} d x\right) \cdot d u_{i-1, j} \wedge d u_{i, j} .
\end{aligned}
$$

Let us introduce two shift operators as

$$
\begin{aligned}
E_{1} f\left(u_{i, j}\right) & =f\left(u_{i+1, j}\right), \\
E_{2} f\left(u_{i, j}\right) & =f\left(u_{i, j+1}\right) .
\end{aligned}
$$

Then the following relations can be found

$$
\begin{aligned}
S_{A\{i, j\}} & =-E_{1} E_{2} S_{E\{i, j\}}, \\
S_{B\{i, j\}} & =-E_{2} S_{D\{i, j\}}, \\
S_{C\{i, j\}} & =-E_{1} S_{F\{i, j\}}, \\
d u_{i, j+1} \wedge d u_{i, j} & =-E_{2}\left(d u_{i, j-1} \wedge d u_{i, j}\right), \\
d u_{i+1, j} \wedge d u_{i, j} & =-E_{1}\left(d u_{i-1, j} \wedge d u_{i, j}\right) .
\end{aligned}
$$


Then it is easy to check that (16) becomes

$$
D_{1} \omega_{D i, j}+D_{2} \tau_{D i, j}=0
$$

where

$$
\begin{array}{r}
\omega_{D i, j}=d u_{i-1, j} \wedge d u_{i, j}-E_{2} S_{E\{i, j\}}-S_{F\{i, j\}}, \\
\tau_{D i, j}=d u_{i, j-1} \wedge d u_{i, j}-S_{B\{i, j\}}-S_{E\{i, j\}}
\end{array}
$$

It is straightforward to show that $\omega_{D}$ and $\tau_{D}$ are two symplectic 2-forms. Namely, they are closed w.r.t. $d$ on the function space and non-degenerate. Then the equation (ए7) is in fact the multisymplectic conservation law. Here $D_{1}$ and $D_{2}$ are given by

$$
D_{1}=E_{1}-1, \quad D_{2}=E_{2}-1
$$

In this letter we have explored some very interesting relations between symplectic and multisymplectic algorithms and the simple finite element method for the boundary value problem of the semi-linear elliptic equation in one-dimensional and twodimensional spaces. The details you could found in [11]. Although what we have found are certain simple boundary value problem of the semi-linear elliptic equation in lower dimensions and also quite simple triangulization in the finite element method, the results still indicate that there should be very deep connections between the symplectic or multisymplectis algorithms and the finite element method.

There are lots of relevant problems should to be studied and some of them are under investigation.

\section{References}

[1] P. G. Ciarlet, The Finite Element Method for Elliptic Problems, North-Holland, Amsterdam, 1978, and references therein.

Selected Works of Feng Kang (I), Ed. by Z.C. Shi et. al. (1994), and references therein.

[2] Selected Works of Feng Kang (II), Ed. by Z.C. Shi et. al. (1995), and references therein.

J.M.Sanz-Serna and M.P.Calvo, Numerical Hamiltonian problem, (1994), Chapman and Hall, London, and references therein.

[3] T.J. Bridges, multisymplectic structures and wave propagations, Math. Proc. Camb. Phil. Soc., 121 (1997)147-190.

T.J. Bridges and S. Reich, multisymplectic integrators: numerical schemes for Hamiltonian PDEs that conserve symplecticity, preprint (1999).

J.E. Marsden, G.W. Patrick and S.Shkoller, Multisymplectic geometry, variational integrators, and nonlinear PDEs, Commun. Math. Phys., 199 (1998) 351-395. 
[4] H.Y. Guo Y.Q. Li and K. Wu, On symplectic and multisymplectic structures and their discrete versions in Lagrangian formalism, ITP-preprint (March, 2001).

[5] H.Y. Guo Y.Q. Li and K. Wu, A note on symplectic algorithms, ITP-preprint (March, 2001).

[6] A.P. Veselov, Integranble discrete-time system and difference operator, Funkts. Anal. Prilozhen., 22 (1988)1-13.

J. Moser and A.P. Veselov, Discrete versions of some classical integrable systems and factorization of matrix polynomials, Commun. Math. Phys., 139 (1991)217243.

[7] J.M. Wendlandt and J.E. Marsden, Mechanical integrators derived from a discrete variation plinciple, Physica D 106 (1997)223-246.

[8] Y.J. Sun and M.Z. Qin, Variational integrators and application for higher order differential equations, CCAST-WL workshop series: 118, 45-58.

[9] H.Y. Guo, K.Wu, S.H. Wang, S.K. Wang and G.M.Wei, Noncommutative Differential Calculas Approach to Symplectic Algorithm on Regular Lattice, Comm. Theor. Phys., 34 (2000) 307-318.

[10] H.Y.Guo, K.Wu and W.Zhang, Noncommutative Differential Calculus on Abelian Groups and Its Applications, Comm.Theor. Phys., 34 (2000) 245-250.

[11] H.Y.Guo, X.M. Ji, Y.Q. Li and K.Wu, On the symplectic and multisymplectic structures in simple finite element method, ITP-preprint, March 2001. 